\title{
High power DFB laser diodes for gas sensing in rough environments
}

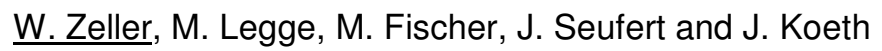

nanoplus Nanosystems and Technologies $\mathrm{GmbH}$

Oberer Kirschberg 4, 97218 Gerbrunn, Germany

Recent years have seen a considerable increase of laser based gas and liquid sensing including fields of application in medical analytics, environmental monitoring or process control.

The significant success of tunable laser diode spectroscopy (TDLS) in these areas is based particularly on its sophisticated detection limits in the range of ppm to ppb [1]. Suitable lasers are an essential prerequisite of this technological approach. Typical devices for example used for $\mathrm{H}_{2} \mathrm{O}$ or $\mathrm{OH}$ sensing operate at emission wavelengths in the $2.6-2.7 \mu \mathrm{m}$ range due to the very intense absorption features of both water and hydroxide in this wavelength range. Such laser diodes have typical output power levels of 1-3 $\mathrm{mW}$ [2].

In harsh environments characterized e.g. by high dust loads however, the level of particle contamination leads to substantial loss in signal due to Rayleigh-scattering of the light. In this case, TDLS application requirements include a high laser output power. Therefore it can be advisable to trade more intense absorption features for higher laser output power and use corresponding laser diodes at shorter emission wavelengths.

We fabricated longitudinally single mode distributed feedback (DFB) laser diodes with continuous wave (cW) output power levels of more than $150 \mathrm{~mW}$ at an emission wavelength of $982 \mathrm{~nm}$.

Applying the DFB concept by defining metal gratings laterally to the ridge waveguide (RWG), longitudinally single mode emission can be achieved without the need for any epitaxial overgrowth. This technique can be applied to a wide range of different material systems [3]. In this case, the RWGs were defined by optical lithography and etched close to the upper waveguide using an electron cyclotron resonance reactive-ion etching (ECR-RIE) process with an $\mathrm{Ar}-\mathrm{Cl}_{2}$ gas-mixture.

Using high resolution electron beam lithography and a lift-off process, first order $\mathrm{Cr}$ gratings were defined laterally to the RWGs. Afterwards, the structure was planarized and insulated with benzocyclobutene $(B C B)$, followed by the evaporation, optical definition and etching of the metallic $p$-contact.

In order to reduce thermal roll-over often limiting achievable output power the contacts were optimized for epi-side down mounting. In a backside lapping step the sample thickness was decreased to $150 \mu \mathrm{m}$ in order to provide better heat dissipation. Then the metallic $\mathrm{n}$-contact was applied and annealed at $420^{\circ} \mathrm{C}$. After cleaving laser bars, anti- and high-reflection coatings with $5 \%$ and $90 \%$ reflectivity were applied to the laser facets. Finally the lasers were mounted epi-side down on c-mounts.

An exemplary emission spectrum of one such laser diode is shown in Fig. 1. The high side mode suppression ratio (SMSR) of $49 \mathrm{~dB}$ at an operating termperature of $40^{\circ} \mathrm{C}$ ensures high spectral selectivity during gas sensing. Light output power characteristics of the same device are depicted in Fig. 2. They were measured in cw mode at temperatures of 25 and $45{ }^{\circ} \mathrm{C}$. 


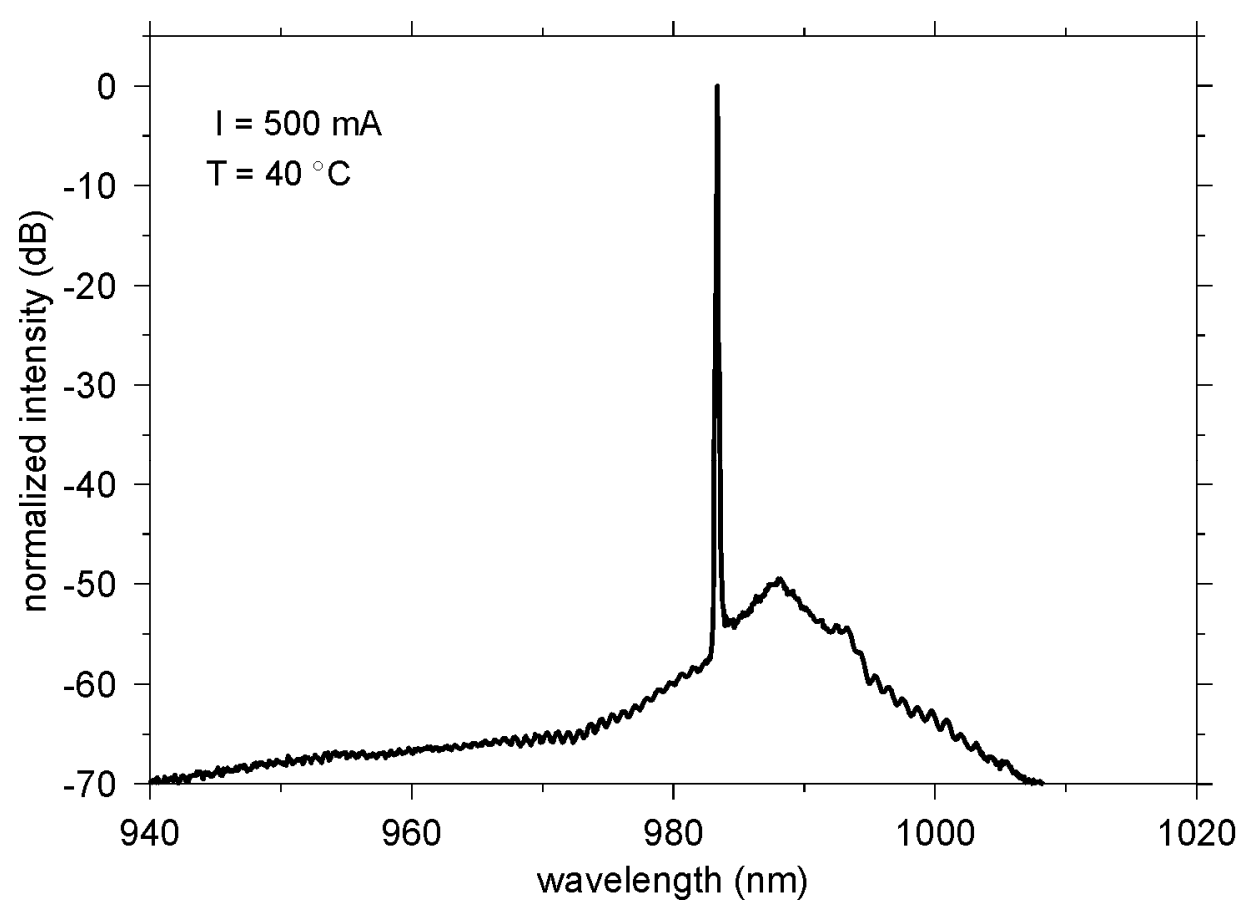

Fig. 1: Emission spectrum recorded at a drive current of $500 \mathrm{~mA}$ and a temperature of $40{ }^{\circ} \mathrm{C}$, showing a SMSR of $49 \mathrm{~dB}$.

Both threshold current and slope efficiency exhibit only small changes under temperature variation. The maximum cw output power level of $175 \mathrm{~mW}$ is higher than those of lasers typically used for water vapor or $\mathrm{OH}$ sensing operating in the $2.6-2.7 \mu \mathrm{m}$ wavelength range by a factor of approximately $50-100$.

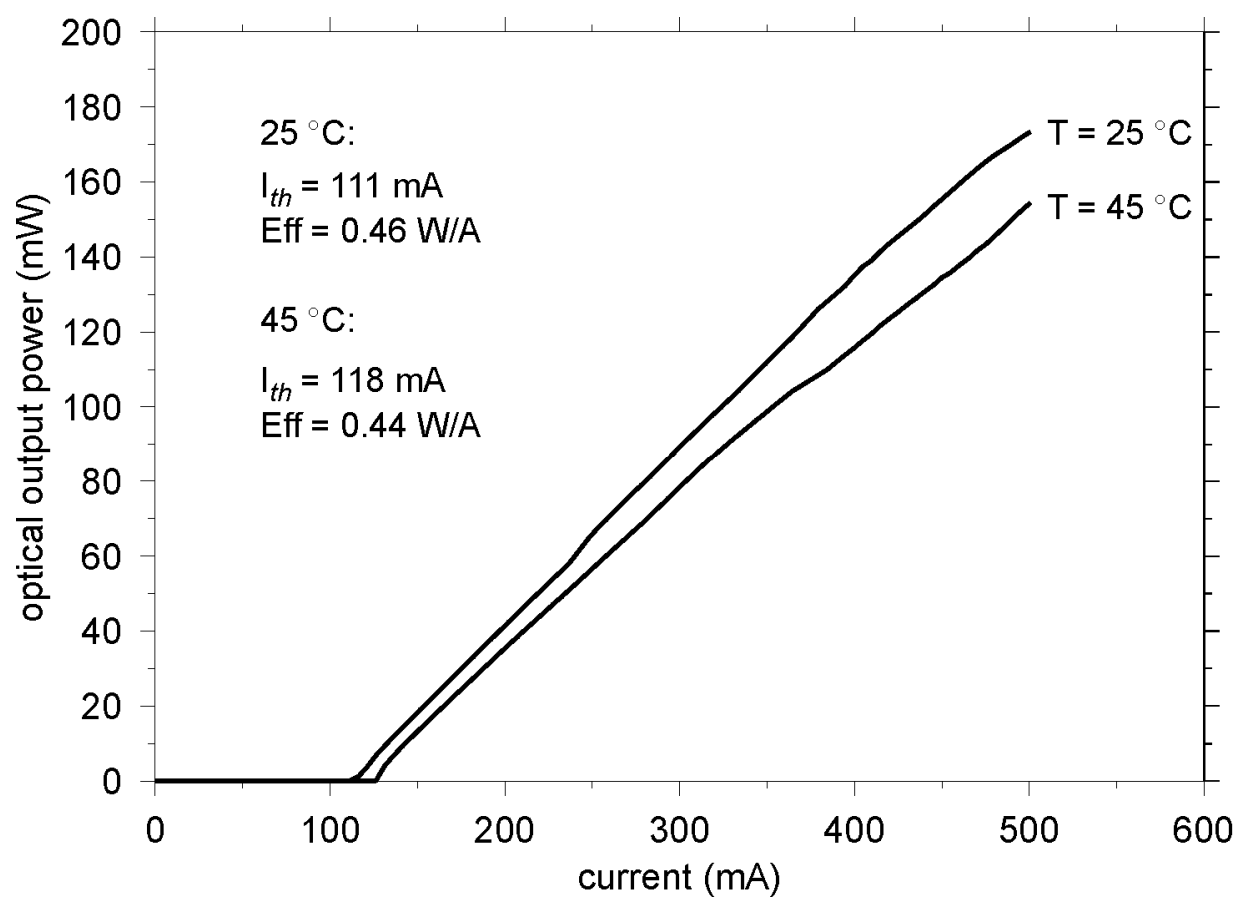

Fig. 2: Light output characteristics recorded at $25^{\circ} \mathrm{C}$ and $45{ }^{\circ} \mathrm{C}$. 
Fig. 3 shows the current induced wavelength tuning characteristic obtained at an operating temperature of $40{ }^{\circ} \mathrm{C}$. All datapoints in the figure correspond to a SMSR of at least $44 \mathrm{~dB}$. By adjusting the drive current of the laser diode, the emission wavelength can be tuned over a range of $1.3 \mathrm{~nm}$. Due to the episide down mounting of the laser diodes, a relatively small current dependency $\mathrm{d} / \mathrm{dT}$ of $0.004 \mathrm{~nm} / \mathrm{K}$ is observed.

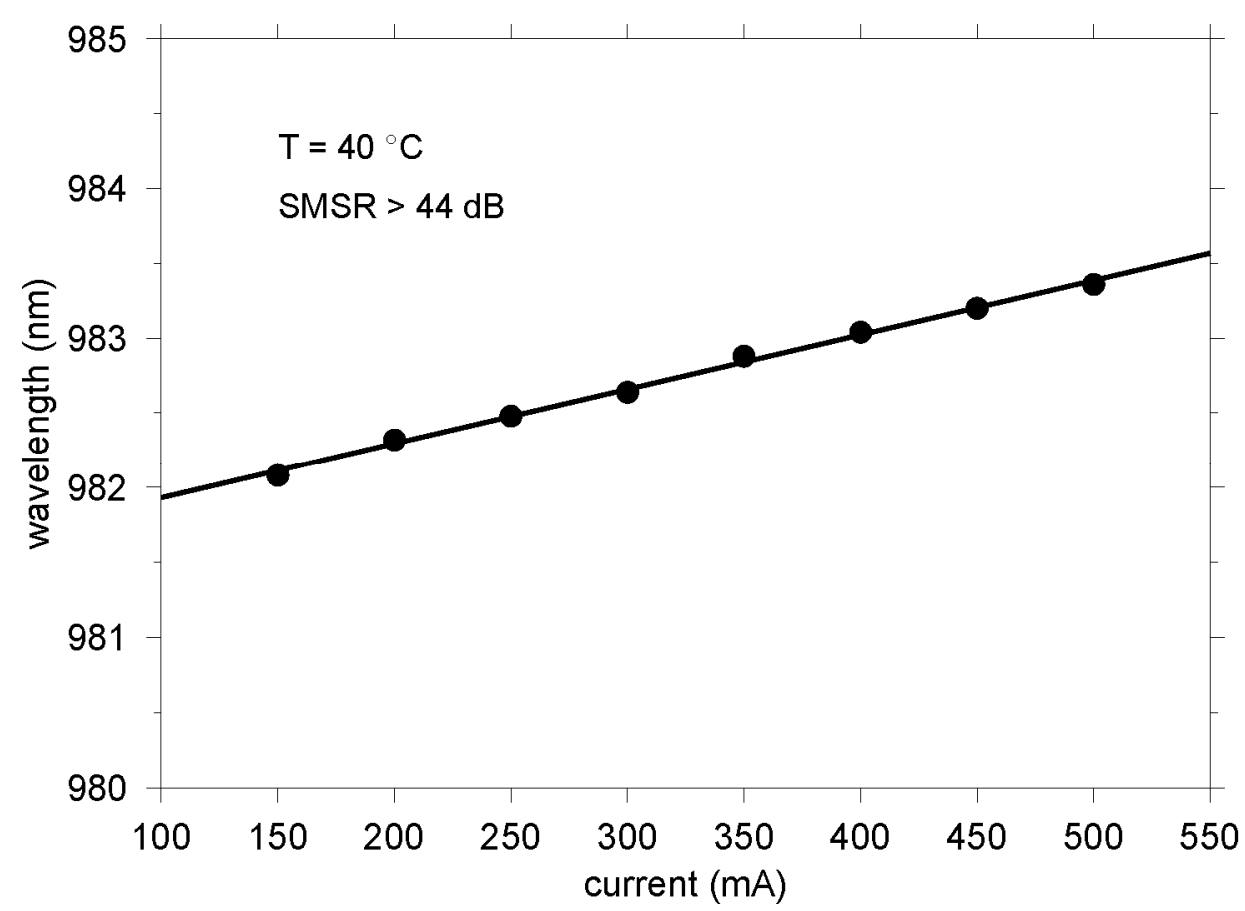

Fig. 3: Wavelength vs drive current measured at a temperature of $40{ }^{\circ} \mathrm{C}$. A wavelength range of $1.3 \mathrm{~nm}$ is covered with SMSR > $44 \mathrm{~dB}$.

Laser diodes operating in the wavelength range around $980 \mathrm{~nm}$ can be used to scan e.g. for $\mathrm{H}_{2} \mathrm{O}$ vapor and $\mathrm{OH}$. Both gas features of course exhibit significantly more intense absorption lines at wavelengths between 2.6 and $2.7 \mu \mathrm{m}$. However laser diodes operating at such long wavelengths are usually limited to optical output powers in the range of a few $\mathrm{mW}$. In contrast, the DFB laser diodes presented here are operating with output powers approximately 50 to 100 times higher, therefore enabling the use of TDLS in environments where scattering losses are a problem.

\section{References}

[1] P. Werle "Diode Laser Sensors for In-Situ Gas Analysis" in P. Hering, J. P. Lay, and S. Stry (Eds.) Laser in Environmental and Life Sciences - Modern Analytical Methods, Springer, Heidelberg, pp. 223 $243(2004)$

[2] J. Seufert, M. Fischer, M. Legge, J. Koeth, R. Werner, M. Kamp, and A. Forchel: "DFB laser diodes in the wavelength range from $760 \mathrm{~nm}$ to $2.5 \mu \mathrm{m}$," Spectrochim. Acta A60, pp.3243-3247 (2004)

[3] M. Kamp, J. Hofmann, A. Forchel, F. Schäfer, and J. P. Reithmaier: "Low threshold high quantum efficiency laterally gain coupled InGaAs/AIGaAs distributed feedback lasers," Appl. Phys. Lett. 74, pp. 483 -485 (1999) 